\title{
Comparison of fecal and cecal microbiotas reveals qualitative similarities but quantitative differences
}

Dragana Stanley ${ }^{1,2,3}$, Mark S Geier ${ }^{3,4,5,6}$, Honglei Chen ${ }^{2}$, Robert J Hughes ${ }^{3,4,5}$ and Robert J Moore $2,3,7^{*}$

\begin{abstract}
Background: The majority of chicken microbiota studies have used the ceca as a sampling site due to the specific role of ceca in chicken productivity, health and wellbeing. However, sampling from ceca and other gastrointestinal tract sections requires the bird to be sacrificed. In contrast, fecal sampling does not require sacrifice and thus allows the same bird to be sampled repeatedly over time. This is a more meaningful and preferred way of sampling as the same animals can be monitored and tracked for temporal studies. The commonly used practice of selecting a subset of birds at each time-point for sacrifice and sampling introduces added variability due to the known animal to animal variation in microbiota.

Results: Cecal samples and fecal samples via cloacal swab were collected from 163 birds across 3 replicate trials. DNA was extracted and 165 rRNA gene sequences amplified and pyrosequenced to determine and compare the phylogenetic profile of the microbiota within each sample. The fecal and cecal samples were investigated to determine to what extent the microbiota found in fecal samples represented the microbiota of the ceca.

It was found that $88.55 \%$ of all operational taxonomic units (OTUs), containing $99.25 \%$ of all sequences, were shared between the two sample types, with OTUs unique for each sample type found to be very rare. There was a positive correlation between cecal and fecal abundance in the shared sequences, however the two communities differed significantly in community structure, represented as either alpha or beta diversity. The microbial populations present within the paired ceca of individual birds were also compared and shown to be similar.

Conclusions: Fecal sample analysis captures a large percentage of the microbial diversity present in the ceca. However, the qualitative similarities in OTU presence are not a good representation of the proportions of OTUs within the microbiota from each sampling site. The fecal microbiota is qualitatively similar to cecal microbiota but quantitatively different. Fecal samples can be effectively used to detect some shifts and responses of cecal microbiota.
\end{abstract}

Keywords: Intestinal, Microbiota, Chicken, Fecal, Cecal

\section{Background}

The avian ceca generally have a more important role in digestion than the cecum in most mammals. Avian ceca are usually finger-shaped blind pouches, presenting as lateral extensions at the junction of the small and large intestine and are commonly present in pairs. In birds the ceca vary considerably in size and morphology; ranging from very long, such as in most domestic poultry, to

\footnotetext{
* Correspondence: rob.moore@rmit.edu.au

${ }^{2}$ Australian Animal Health Laboratory, CSIRO Animal, Food and Health Sciences, Geelong, VIC 3220, Australia

${ }^{3}$ RMIT University, Poultry Cooperative Research Centre, University of New England Armidale, New South Wales 2315, Australia

Full list of author information is available at the end of the article
}

completely absent, such as in pigeons and parrots [1]. In the chicken they reach $16-18 \mathrm{~cm}$ long in adult birds. It is a multi-purpose organ vital to the bird's physiology; a complex system inhabited by a very dense microbial community that converts the cecal pouches into fermentation powerhouses. Members of the cecal microbiota have the ability to digest cellulose, starch and other resistant polysaccharides [1,2]. Ceca are not only a major site of water absorption $[1,3]$ but are also a site of nutrient transport and absorption [4].

Recent advances in culture-free technologies for microbiota characterization have facilitated an increasing number of studies investigating changes in chicken microbiota within the gastrointestinal tract (GIT) following 
manipulation of feed, environment, or health. It has been documented that chicken microbiota responds to changes in feed [5-7], litter composition [8], antibiotics [9] and probiotic addition to feed $[10,11]$, disease $[12,13]$ and stress $[14,15]$. Recently the use of next generation sequencing techniques to study microbiota composition has been extended beyond phylogenetic analysis to also include functional analysis using metagenomics of whole cecal microbiotas [16].

The important biological role of the cecum has resulted in it being a major focus of research into the influences of chicken microbiota on bird health and productivity [13,17-19]. The ceca sample microbes from both descending and ascending microbiota via normal peristalsis and retrograde gut movements $[20,21]$. The cecal content is emptied several times per day into the gut lumen and then regrows to fill the ceca. This cyclic emptying of the ceca means that elements of the fecal microbiota must be directly derived from the ceca. We were interested to examine the relationship between the population structure of cecal and fecal microbiotas to determine if fecal sampling via cloacal swabbing is an effective proxy for cecal sampling. Cloacal sampling, which is effectively a reliable and fast method of collecting fresh fecal samples from a specific bird, has the great advantage that it is easy to obtain a series of samples from the one bird over time, unlike the case with cecal samples where it is usual to sacrifice the bird to recover a sample and so only a single time-point snapshot can be obtained for any one bird. This has meant that temporal studies of cecal microbiota have had to rely on population sampling with different birds studied at each time point. The analysis of such data is complicated by the large inter-bird variation in microbiota structure that has recently been documented [22]. If the relationship between fecal and cecal samples was understood and consistent then fecal samples could be used to determine at least some elements of the microbiota present in the ceca.

The fecal and cecal microbiota profiles of mice have been compared using a gel based analysis method and no obvious correlations were noted [23] however the biology of the chicken ceca is very different to that of the mouse and so it is likely that this study gives little indication of the relationship of the two sites in chickens. Sekelja et al. [24] used conventional sequencing of cloned 16S fragments to investigate the variability of chicken fecal microbiota and how this was related to emptying of different regions of the GIT. Here we report a large chicken study that used next generation sequencing technology to characterize GIT microbiota. The study builds on previously reported work that analyzed the cecal microbiota of birds in a series of production efficiency trials [22]. Here we analyse further samples and extend the microbiota analysis to generate a statistically powerful data set to compare fecal and cecal sample pairs from 163 birds to determine the level and nature of similarity in microbiota structure between the two sample sites. The relationship between the microbiota in the two cecal compartments within each bird was also investigated.

\section{Results}

Cecal microbiota is richer in OTUs and has fewer dominant OTUs compared to fecal microbiota

A total of $1,770,812$ sequences were obtained from the 326 samples included in this analysis (163 birds with adequate recovery of sequences from both cecal and fecal samples). After quality trimming and chimera checking $1,076,820$ sequences remained with a minimum length of 324 nucleotides, a maximum length of 600 , and an average read length of 507 nucleotides. There were on average over 3,300 sequences per sample.

A number of alpha diversity measures were inspected to compare within sample diversity of cecal and fecal samples. All of the diversity indicators showed statistically significant differences, all with $\mathrm{P}<1 \mathrm{e}^{-5}$, the lowest possible $\mathrm{p}$-value based on the $1 \mathrm{e}^{5}$ Monte Carlo permutations used (Qiime). Both non-phylogenetic estimator Chao1 (Figure 1A) and phylogenetic diversity (PD) indicator whole tree analysis showed higher diversity in cecal samples relative to fecal samples. Cecal richness, based on number of observed species, was significantly higher than richness in fecal communities. The singles and doubles estimator suggested more rare OTUs in cecal samples. Simpson's evenness (Figure 1B) and Strong's dominance index confirmed that fecal samples have more dominant OTUs. Shannon entropy (Figure 1C) and the Equitability indices (Figure 1D) showed cecal phylotypes as more evenly distributed within samples.

A range of beta diversity measures were inspected (Jaccard, Bray Curtis, Canberra, Chord, Euclidean, Manhattan, Pearson, Soergel,) and, similar to alpha diversity, they indicated significant differences between the fecal and cecal samples. Unweighted and weighted UniFrac (Figure 2) both showed differences in microbial communities with ADONIS statistics P-values lower than $\mathrm{P}<1 \mathrm{e}^{-5}$, the lowest possible P-value based on the $1 \mathrm{e}^{5}$ permutations. Separation in both weighted and unweighted UniFrac indicated that the microbiota profiles group on origin (fecal or cecal) based on presence/absence as well as abundance.

\section{Members of cecal and fecal microbiota}

Most of the OTUs were present in both cecal and fecal samples (Figure 3). The shared OTUs, represented by $88.55 \%$ of all OTUs, accounted for $99.25 \%$ of all sequences. The $7.4 \%$ of OTUs that were exclusive to 

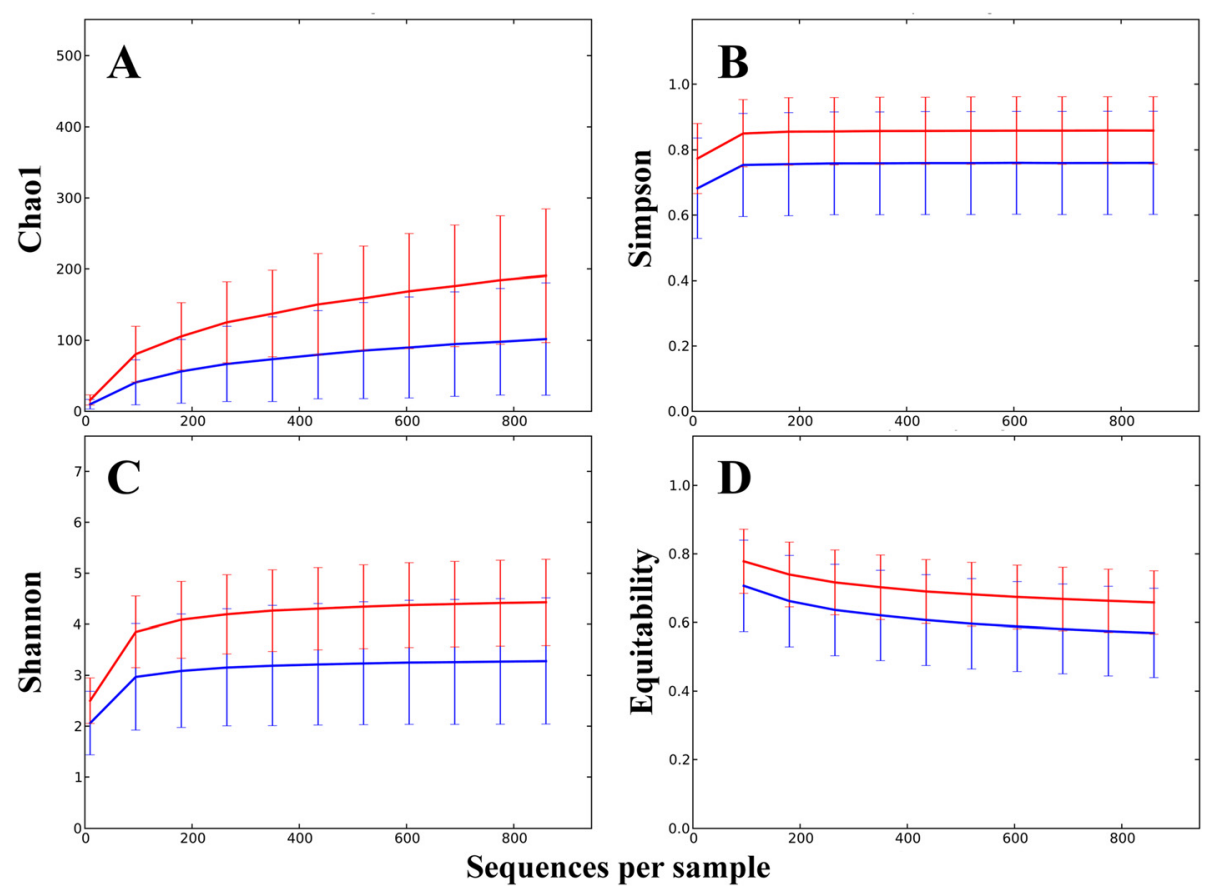

Figure 1 Alpha diversity is significantly different between fecal and cecal microbiota. Alpha diversity indicators of cecal (red) and fecal (blue) samples all showed significant $\left(p<1 e^{-5}\right)$ difference based on $1 e^{5}$ Monte Carlo permutations. The indices plotted in the four panels are; A, Chao 1; B, Simpson; C, Shannon; and D, Equitability.

cecal samples comprised only $0.27 \%$ of all sequences, indicating that they are mostly rare, low abundance OTUs. Fewer OTUs were exclusive to fecal samples (4.05\%), however they made up a greater proportion of all sequences $(0.45 \%)$ than the cecum exclusive OTUs. OTUs present only in cecum were all, with the exception of one OTU, unknown and uncultured relatives of Bacteroides fragilis, Faecalibacterium prausnitzii, and a number of unknown Lactobacillus and clostridia. On the other hand, $27 \%$ of OTUs exclusive to fecal origin shared sequence similarity higher than $97 \%$ to known type strains of bacteria not commonly found in chicken cecum such as Streptococcus minor, Vagococcus fluvialis, Streptococcus henryi, Staphylococcus gallinarum and Staphylococcus aureus, all with 100\% sequence identity to type strains.
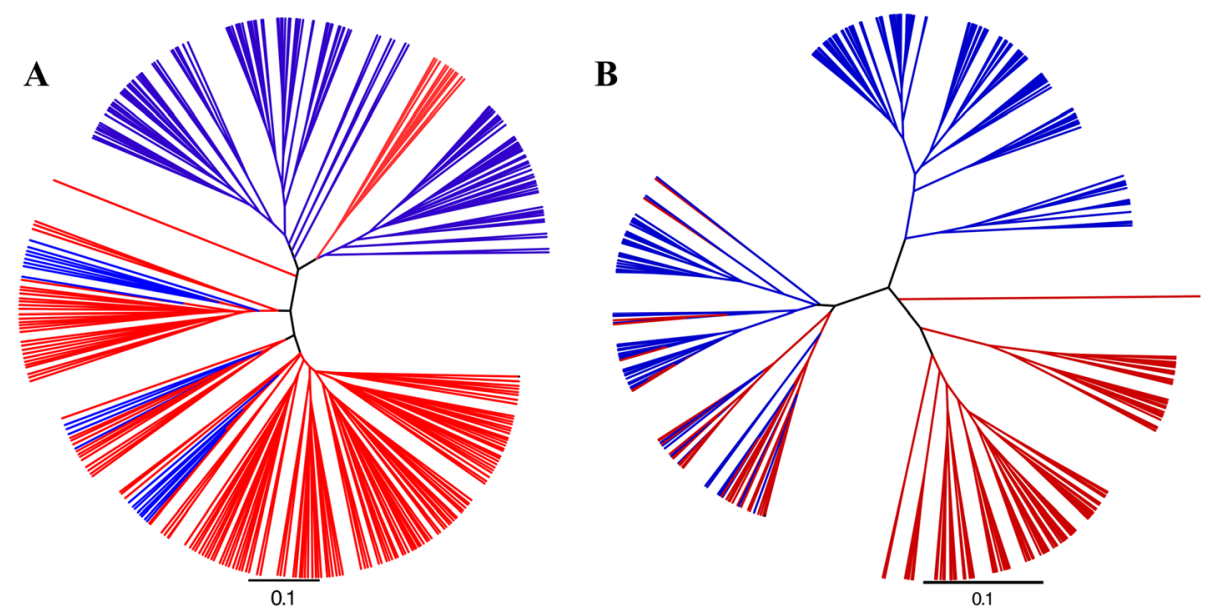

Figure 2 UniFrac distance shows microbiota differences between fecal and cecal origins. A tree representing cecal (red) and fecal (blue) samples. The sample clustering is based on jacknifed weighted (A) and unweighted (B) UniFrac. Tree files were generated in Qiime and visualised in FigTree. The two communities were significantly $\left(p<1 \mathrm{e}^{-5}\right)$ different based on both weighted and unweighted UniFrac and ADONIS statistics using $1 e^{5}$ permutations. 


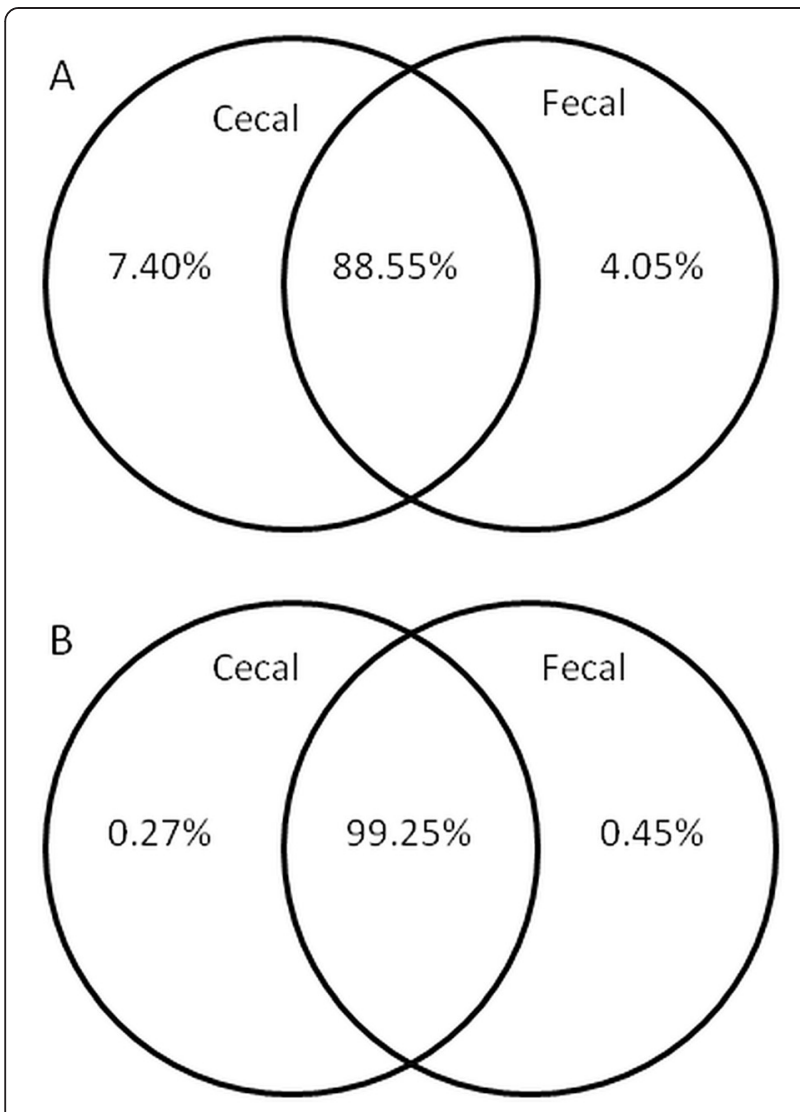

Figure 3 Venn diagrams showing percentage of shared OTUs. Diagrams are show the percentage of shared OTUs (A) and the percentage of sequence reads that they represented (B) of cecal and fecal origin at 3\% divergence. $88.55 \%$ of all OTUs are shared between the sections and those OTUs represented $99.25 \%$ of all sequences.

Tables 1 and 2 show the 20 most abundant OTUs, based on sequence counts, from cecal and cloacal samples respectively, classified using EzTaxon to their nearest culturable isolate. The most abundant OTU in chicken cecum, across the 3 trials, was $99.6 \%$ identical to a type strain of Bacteroides fragilis and represented $14.1 \%$ of all cecal sequences based on the 100-times rarefied data (Table 1). This OTU was the most abundant only in trial 1 with $20.3 \%$, third most abundant in trial 2 (8\%) and second most abundant in trial 3 with $14.4 \%$ of total sequences. The next 4 most abundant OTUs were confidently (>99\% similarity) classified as Lactobacillus crispatus, Lactobacillus johnsonii, Lactobacillus salivarius and Lactobacillus reuteri. There were 3 more OTUs in the cecal top 20 classified with sequence similarity $>97 \%$ to Lactobacillus helveticus, Lactobacillus vaginalis and Parabacteroides distasonis. The remaining OTUs, except for Lactobacillus helveticus (96.6\%) were of unknown species with sequence similarity to the closest known isolates in the range of $91.9-78.3 \%$. Cecal microbiota contained more unknown OTUs (similarity to known isolate $<97 \%$ ) than the fecal microbiota; those OTUs comprised $47 \%$ of cecal microbiota as opposed to $33.2 \%$ in fecal samples, based on total cecal and total fecal sequences.

Fecal microbiota was dominated by Lactobacillus with the 5 most abundant OTUs making up $54.5 \%$ of all fecal sequences. The most abundant classified with $>97 \%$ sequence similarity to type strains were $L$. crispatus, $L$. salivarius, L. johnsonii, L. helveticus, L. reuteri and $L$. vaginalis. Moreover, only 4 out of 20 most abundant OTUs were not Lactobacilli: Bacteroides fragilis, Candidatus Arthromitus, Clostridium perfringens and unknown clostridium similar to $C$. lituseburense. The remaining Lactobacilli OTUs, which showed sequence similarity to the closest culturable isolates of between 89.4 and $94.4 \%$, most likely comprise a number of novel members of this genus. There were 406 OTUs out of 1282 total fecal and cecal, classified as most closely related to Lactobacillus strains, 351 of those with sequence similarity $<95 \%$ to known culturable isolates.

Fecal samples contained Clostridium perfringens at an average level of $4.5 \%$, the $6^{\text {th }}$ most abundant OTU classified, with $99.6 \%$ similarity to the type strain. However, cecal samples contained on average only $0.14 \%$ of this known chicken pathogen. The carriage of C. perfringens varied markedly across the 3 trials; in Trial 2 the fecal samples carried $12.8 \%$, Trial 3 had $0.5 \%$ and none was detected in Trial 1. Although $C$. perfringens carriage was lower in the cecal samples the same relative trend between trials was noted with $0,0.3$ and $0.1 \%$ detected in trials $1-3$ respectively.

\section{Low abundance fecal OTUs more closely correlate with cecal levels than high abundance OTUs}

The main question we aimed to answer in this study was whether fecal samples could provide a reliable snapshot of cecal community structure. The high number of sequences and samples across the three trials provides sufficient statistical power to attempt to make these predictions. The three trials were inspected separately and as a whole set for correlations between fecal and cecal abundance of all shared OTUs present in both cecal and fecal samples across all of the birds. All 3 trials showed positive correlation between cecal and fecal abundances (Figure 4). To inspect if the level of correlation is influenced by abundance, i.e., if more abundant OTUs show better correlation, we inspected correlations for subsets of data with different cecal and separately fecal minimal abundance for all trials. Although we expected that more abundant OTUs would have higher cecal-fecal correlation, we found the opposite trend for both cecal and fecal abundances (Figures 4,5,6). Rare OTUs seem to be of similar low abundance in both cecal and fecal while higher abundance taxa tended to differ more. This is 
Table 1 The 20 most abundant OTUs in chicken cecum

\begin{tabular}{|c|c|c|c|c|c|}
\hline Closest culturable isolate & Isolate accession number & $\%$ similarity & $\%$ in cecal & $\%$ in fecal & OTU ID \\
\hline Bacteroides fragilis & CR626927 & 99.62 & 14.1 & 1.2 & 4 \\
\hline Lactobacillus crispatus & Y17362 & 99.81 & 11.3 & 15.4 & 1 \\
\hline Lactobacillus johnsonii & ACGR01000047 & 99.61 & 9.6 & 12.2 & 2 \\
\hline Lactobacillus salivarius & AF089108 & 99.43 & 5.4 & 12.7 & 3 \\
\hline Lactobacillus reuteri & AP007281 & 99.40 & 4.3 & 5.6 & 5 \\
\hline Acholeplasma palmae & L33734 & 79.53 & 3.9 & 0.5 & 8 \\
\hline Lactobacillus helveticus & ACLM01000202 & 97.62 & 3.5 & 8.6 & 133 \\
\hline Butyricicoccus pullicaecorum & EU410376 & 82.18 & 3.4 & 0.2 & 9 \\
\hline Faecalibacterium prausnitzii & AJ413954 & 94.71 & 2.3 & 0.3 & 12 \\
\hline Lactobacillus vaginalis & AF243177 & 99.60 & 1.3 & 0.8 & 10 \\
\hline Lactobacillus helveticus & ACLM01000202 & 96.67 & 1.2 & 2.7 & 874 \\
\hline Pontibacillus litoralis & EU583724 & 78.46 & 0.9 & 0.2 & 13 \\
\hline Exiguobacterium acetylicum & X70313 & 78.33 & 0.9 & 0.1 & 14 \\
\hline Ruminococcus albus & L76598 & 82.62 & 0.9 & 0.1 & 21 \\
\hline Ruminococcus albus & L76598 & 89.88 & 0.8 & 0.1 & 16 \\
\hline Parabacteroides distasonis & CP000140 & 97.31 & 0.8 & 0.0 & 18 \\
\hline Ruminococcus flavefaciens & X83430 & 86.85 & 0.8 & 0.1 & 15 \\
\hline Clostridium cellobioparum & X71856 & 83.27 & 0.7 & 0.1 & 17 \\
\hline Clostridium termitidis & FR733680 & 80.37 & 0.7 & 0.1 & 23 \\
\hline Clostridium symbiosum & M59112 & 91.97 & 0.7 & 0.1 & 25 \\
\hline
\end{tabular}

The representative sequences for all abundant OTUs are publically available in EMBL database with OTU ID as identifier with accession numbers HG810851- HG810882.

especially notable in the fecal dominant OTUs. OTUs comprising more than $50 \%$ of cecal sequences in one bird (Figure 5A) had a wide range of fecal abundances. The number of birds with cecal microbiota dominated by more than $50 \%$ was much lower than in fecal samples. Figure 6 simplifies the message from detailed Figure 5A and B; shared OTUs present in lower abundance correlated better, while correlation for more abundant OTUs was very low.

\section{The microbiota of cecal pairs are similar}

The interpretation of cecal microbiota data could be influenced by sampling procedure if the pair of cecal pouches within a bird had radically different microbiota populations. To address this issue, in an independent analysis, each of the pair of ceca within 24 birds were sampled and analysed according to the same methods detailed for the main experiment. There was no statistically significant difference in alpha diversity between cecal pairs with Shannon, Simpson or Observed Species indices (P-values of $0.98,0.77$ and 0.56 respectively). Beta diversity was also unaffected by choice of ceca; Unweighted UniFrac differences using ADONIS statistics showed a P-value of 0.81 and Unweighted Unifrac P-value of 0.72. In Figure 7 it can be seen that the pairs of cecal samples from each bird tend to be closely related to each other and hence map close to each other in both the unweighted (Figure 7A) and weighted (Figure 7B) UniFrac plots.

\section{Discussion}

Chicken cecal microbiota has been widely investigated due to the significant role of the cecum in heath, performance, and disease $[1,2]$. The first insights into cecal microbiota that suggested the complexity of the microbial populations came from culture-based studies. It has been suggested that the microbial diversity of chicken cecum has been altered with the common use of antibiotics in feed worldwide and that it has lost much of its natural chicken specialised microbiota and with it microbial potential and metabolic capabilities [1,25].

Ceca have been found to harbour microbiota capable of degrading cellulose and other indigestible carbohydrates and producing high amounts of beneficial metabolites such as short chain fatty acids (SCFA) [1]. Along the GIT the cecal community takes the most time to develop and mature [2] and it contains the highest microbial diversity within the GIT [13,26-29]. Although it was reported that differences between trials and flocks in chickens can be extensive, overall cecal composition identified in the present study agrees with previously published data. Lu et al. [28] found clostridia to 
Table 2 The $\mathbf{2 0}$ most abundant OTUs in chicken feces

\begin{tabular}{|c|c|c|c|c|c|}
\hline Closest culturable isolate & Isolate accession number & $\%$ similarity & $\%$ in cecal & $\%$ in fecal & OTU ID \\
\hline Lactobacillus crispatus & Y17362 & 99.81 & 11.3 & 15.4 & 1 \\
\hline Lactobacillus salivarius & AF089108 & 99.43 & 5.4 & 12.7 & 3 \\
\hline Lactobacillus johnsonii & ACGR01000047 & 99.61 & 9.6 & 12.2 & 2 \\
\hline Lactobacillus helveticus & ACLM01000202 & 97.62 & 3.5 & 8.6 & 133 \\
\hline Lactobacillus reuteri JC & AP007281 & 99.40 & 4.3 & 5.6 & 5 \\
\hline Clostridium perfringens & СР000246 & 99.60 & 0.1 & 4.5 & 6 \\
\hline Lactobacillus crispatus & Y17362 & 91.98 & 0.3 & 2.9 & 2234 \\
\hline Clostridium lituseburense & M59107 & 96.91 & 0.4 & 2.8 & 7 \\
\hline Lactobacillus salivarius & AF089108 & 93.01 & 0.3 & 2.8 & 2200 \\
\hline Lactobacillus helveticus & ACLM01000202 & 96.67 & 1.2 & 2.7 & 874 \\
\hline Candidatus Arthromitus sp. & X80834 & 100.00 & 0.0 & 2.1 & 11 \\
\hline Lactobacillus salivarius & AF089108 & 94.44 & 0.1 & 2.1 & 1905 \\
\hline Lactobacillus crispatus & Y17362 & 94.50 & 0.1 & 1.2 & 1845 \\
\hline Lactobacillus johnsonii & ACGR01000047 & 89.41 & 0.2 & 1.2 & 2251 \\
\hline Bacteroides fragilis & CR626927 & 99.62 & 14.1 & 1.2 & 4 \\
\hline Lactobacillus pontis & AJ422032 & 92.01 & 0.1 & 1.0 & 2154 \\
\hline Lactobacillus helveticus & ACLM01000202 & 98.59 & 0.2 & 0.9 & 254 \\
\hline Lactobacillus vaginalis & AF243177 & 99.60 & 1.3 & 0.8 & 10 \\
\hline Lactobacillus crispatus & Y17362 & 93.60 & 0.1 & 0.7 & 1557 \\
\hline Lactobacillus gallinarum & AJ417737 & 90.77 & 0.1 & 0.6 & 1814 \\
\hline
\end{tabular}

The representative sequences for all abundant OTUs are publically available in EMBL database with OTU ID as identifier with accession numbers HG810851- HG810882.

dominate chicken cecum in their trial. In the present study 8 of the 20 most abundant OTUs, based on sequence counts and not adjusting for rRNA operon copy number, are most closely related to the Clostridiales order. However all of these had $<95 \%$ sequence similarity to known culturable isolates, indicating that there is a high degree of cecal microbiota diversity that is yet to be revealed. In general the clostridia have a reputation as bad, undesirable members of the microbiota, based on pathogenic potential of strains of C. perfringens, C. difficile, C. tetani and a few others. However, this order also encompasses many beneficial bacteria such as cellulose and starch degraders; Clostridium clusters IV and XIV have been linked to prevention of inflammatory bowel disease (IBD) and maintenance of mucosal homeostasis [30,31] and clostridia protect from allergy and autoimmune disorders [32] in mice. Atarashi et. al. [32] reported the colonisation of germ free mice with a mix of Clostridium strains and also inspected mice enriched in Clostridium abundance. Clostridium strains promoted $\mathrm{T}_{\text {reg }}$ cell accumulation. Oral administration to conventionally grown mice, to increase Clostridia abundance, was beneficial for mice health, for example in inducing resistance to colitis by the means of suppressing weight loss, bleeding, colon shortening, edema and other symptoms of colitis allowing Clostridia supplemented mice to show mild, if any, symptoms. Chicken cecum is a major source of uncultured Clostridia that may represent enormous microbial potential.

The abundance of Bacteroides, Lactobacillus and Clostridiales in the cecum in the present study is in broad agreement with previous studies [18,19,26-29,33,34]. The benefits of Lactobacillus are well known, cecal samples in this study have shown high diversity in this genus and indicated potential for further probiotic research by estimating 351 potentially novel Lactobacillus-related species with similarity to the closest Lactobacillus database matches of $<95 \%$.

The sequence count data indicate that the most abundant OTU in the cecal microbiota was B. fragilis due to extreme values in one of the trials. Bacteroides are known to have beneficial effects on the host, being effective degraders of indigestible carbohydrates, especially cellulose and starch [35]. B. fragilis produces SCFA [36] and when colonising germ free animals aids $\mathrm{T}_{\text {reg }}$ differentiation and IL10 production [37]. Similarly another abundant phylotype related to Faecalibacterium prausnitzii belongs to a butyrate producing cluster [38]. Absence of Faecalibacterium prausnitzii is linked to Crohn's disease [30] while cecal richness in Ruminococcus is in line with the previously reported [1] cellulose degrading potential [39] of the ceca. 


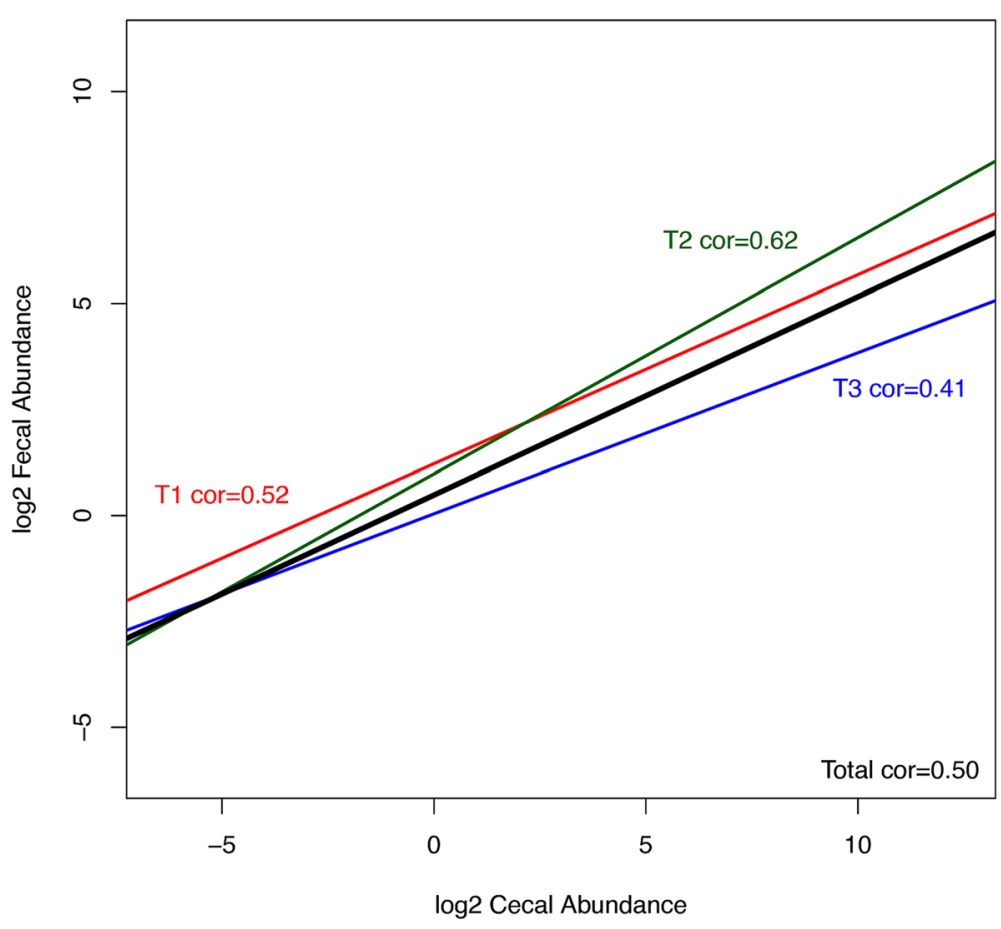

Figure 4 Correlations between cecal and fecal abundance. Line graph representing correlations between cecal and fecal abundance in trial 1 (red), trial 2 (green), trial 3 (blue) and complete set (black line). There is a reproducible positive correlation across the 3 trials. All individual OTUs present in both cecal and fecal sample for all 163 birds were used in calculation, however, due to a very high number they are not displayed in the plot.

Fecal microbiota was found to be dominated by Lactobacillus. This is consistent with previous studies [40]. The high abundance of $C$. perfringens was attributed to one trial with extreme values and $B$. fragilis was present but at much lower abundance than in the cecum. Surprisingly we identified an abundant fecal OTU, comprising $2.1 \%$ of fecal sequences, as $100 \%$ identical to Candidatus Arthromitus sp. LSFO1.94,LSFO2.94
(EzTaxon database type strain). Gong et al. [33] reported high abundance of Candidatus division Arthromitus in chicken jejunum and ileum mucosal samples, representing $34 \%$ and $28 \%$ of sequences in these regions respectively. They are found in gut microbiota of humans, chicken, rodents and fish, where they anchor to the intestinal epithelial cells in the ileum to act as immune system modulators [41]. They are major activators of T-cells [42,43],
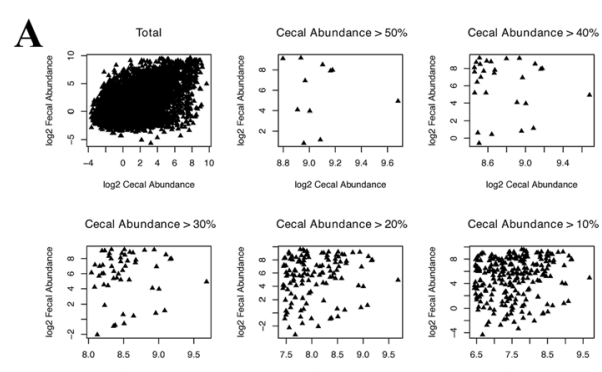

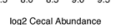
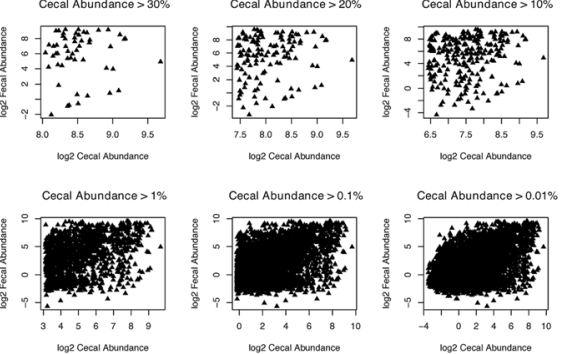
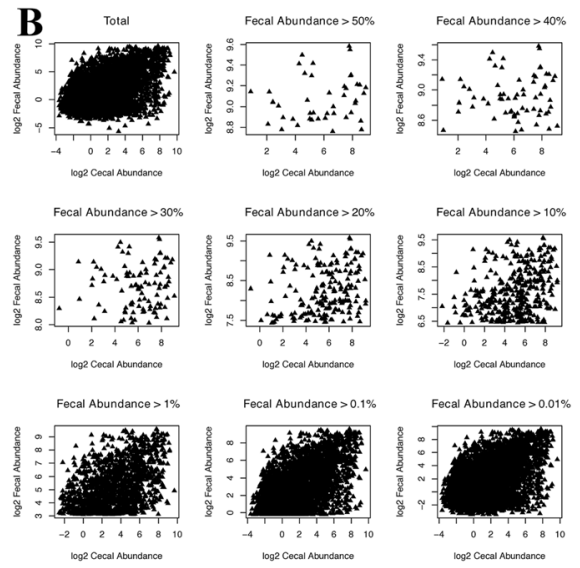

Figure 5 Correlation plots for the different minimal cecal (A) and fecal (B) abundances. Note that each shared OTU was plotted for all 163 birds and may be highly abundant in many of the birds, thus total percentages are not expected to add up to 100 . For example, there were 11 birds that had OTUs with cecal abundance higher than $50 \%$ vs 43 birds that contained fecal OTUs with abundance over $50 \%$. 


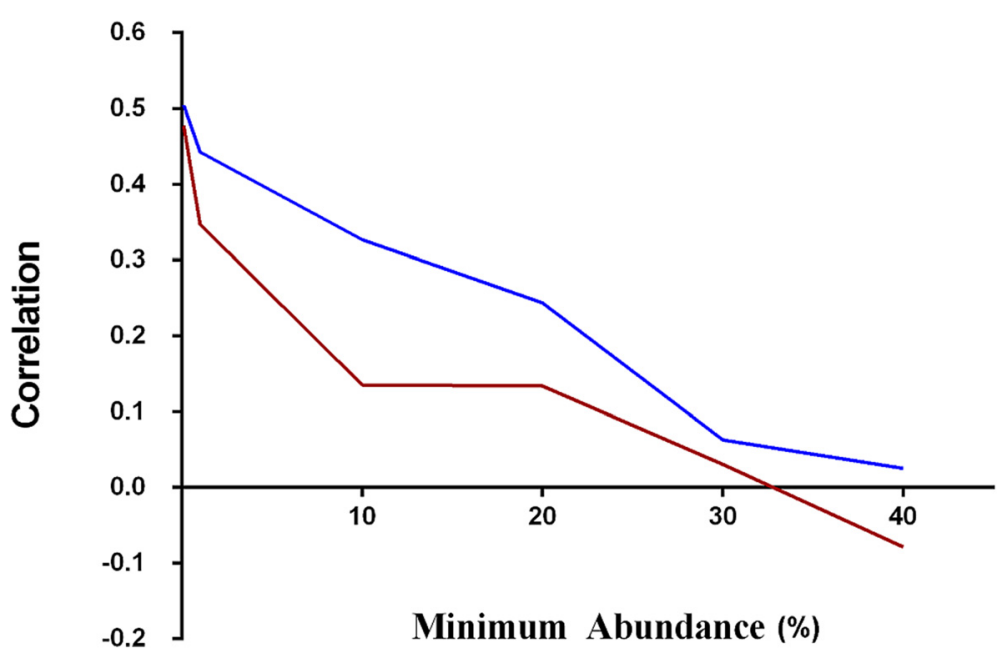

Figure 6 Relationship between fecal/cecal correlation and levels of OTU abundance. Correlations were plotted for both cecal (red) and fecal (blue) samples across the range of OTU abundances. There was linear relationship between correlation and minimum abundance (\%) showing higher correlation for low abundance OTUs and a very low correlation for high abundance OTUs.

epithelial lymphocytes, [42,44] and IgA [45] and could thus also be important in chicken health and performance. Until recently Candidatus Arthromitus was the term used for two physiologically similar groups of segmented filamentous bacteria (SFB), one commonly found in the gut of arthropods and others commonly found in GIT of vertebrates [46]. Although there were striking similarities between the two groups, Thompson et al. [46] demonstrated them to be distinct and unrelated. Arthropod inhabiting filamentous bacteria have yet unknown function while the ones inhabiting GIT of vertebrates, including chicken, play absolutely critical roles in immune function of the host [47]. Thompson et al. suggested that GIT originating SFB form a monophyletic group in the Clostridiaceae based on $16 \mathrm{~S}$ sequence analysis, are now renamed "Candidatus Savagella" [46]. However, arthropods specific filamentous bacteria were identified as members of a Lachnospiraceae Arthromitus cluster and should keep the name "Candidatus Arthromitus" [46]. The abundance of Candidatus Savagella in chicken fecal microbiota is not reproducible across the

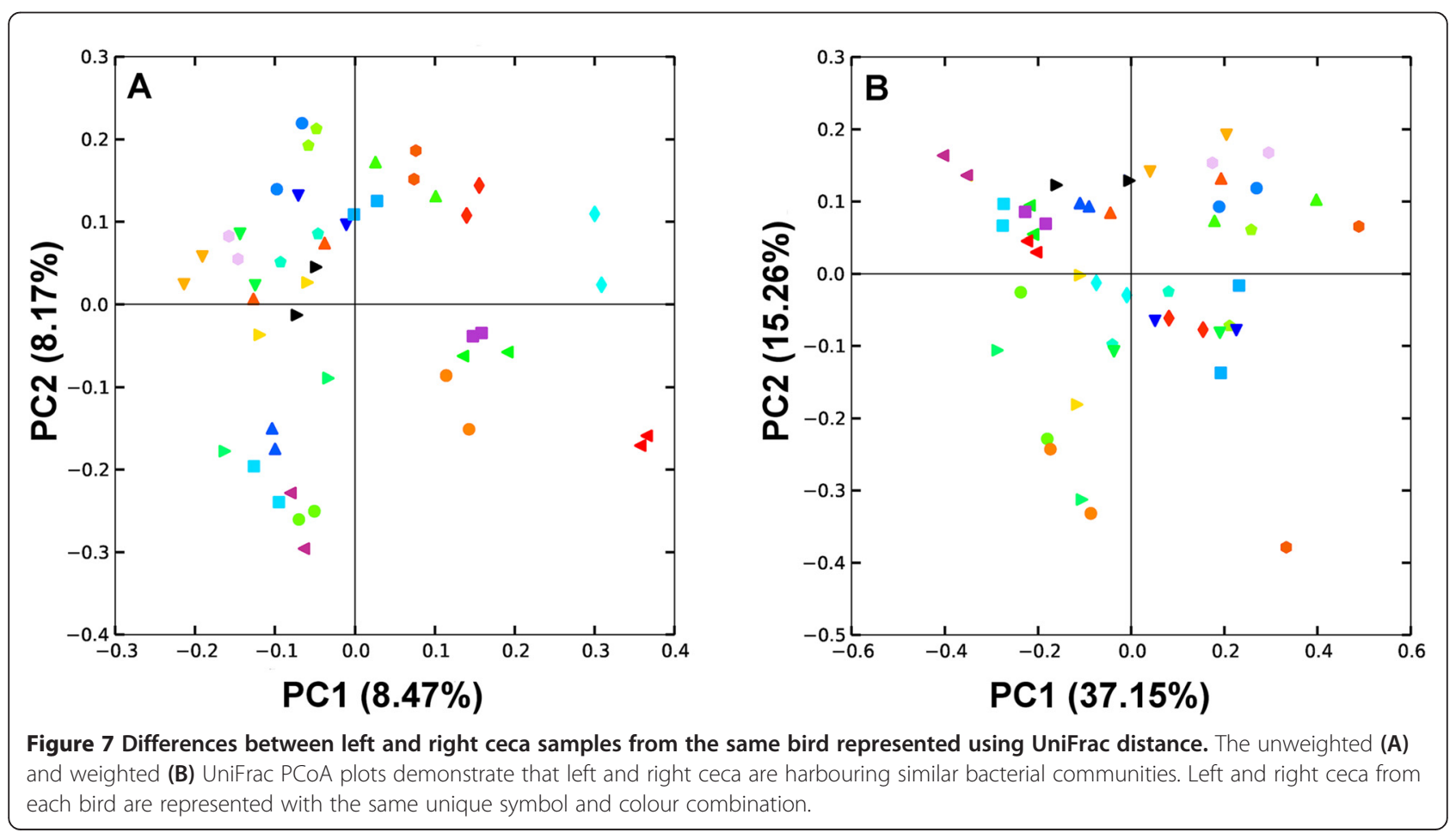


studies. In our own opinion its identification is influenced by the taxonomic databases and algorithms used and in many cases they fall under unknown and uncultured bacteria. It is possible that their role in chicken health, especially immunity is significant therefore more attention should be given to deeper taxonomic identification of significant OTUs in chicken studies.

Sekelja et al. [24] inspected the influence of other GIT sections on chicken fecal microbiota over 16 days. They detected massive temporal variations in fecal microbiota and different profiles to match different sections of GIT. They proposed that the major reason for the temporal variation was periodic emptying of different GIT sections. The emptying of different GIT areas may influence fecal profile but fecal analysis still remains a useful and powerful approach for microbiota studies in animals with proven success record in human and mammalian studies. It is possible that the timing of sampling and cecal emptying is the reason for the existence of a number of outlier birds with higher similarity between fecal and cecal communities. In an auxiliary study we found that there were strong similarities between the microbiota compositions of pairs of ceca from birds, indicating that the choice of ceca is unlikely to be a concern in sampling procedures. Perhaps a major lesson to be learnt is that it may be misleading to draw conclusions from just a few samples; a large number of samples will represent a range of different emptying events from different regions of the GIT and may allow a more complete and representative overall picture to emerge of microbiota composition.

In the present study we compared fecal and cecal microbiota across many birds and three independent trials in order to investigate the relationship between the two most commonly used and reported sample types for microbiota analysis in birds. We were particularly interested in determining the extent to which the fecal microbiota may reflect the content of the cecal microbiota. From this extensive sample set we can conclude that for the majority of OTUs (88.55\%), comprising $99.25 \%$ of sequences, presence in fecal implies presence in cecal community.

Our results show the highest correlation between fecal and cecal samples within the rare biosphere species, the correlation analysis however, included only shared OTUs, but all OTUs that were not shared (i.e., present only in fecal or only in cecal samples) were also from the low abundance group. Therefore, high correlation among rare OTUs cannot be used to exactly predict cecal from fecal abundance since some rare OTUs are also likely not to be detected in the other community at all. The data shows that OTUs exclusive to cecum, which would be missed if fecal origin was sampled instead, were all of low abundance.
Based on the positive correlation for nearly all shared OTUs it is possible that the low abundance OTUs that appear to be unique to the cecal or to fecal samples would be identified in the opposite group if the depth of sequencing was higher as they are near the detection limit and the limits of the exclusion criteria used in quality filtering parameters to generate the data set. Although the alpha diversity plots indicated satisfactory sequence coverage an increase in sequencing depth may identify more shared OTUs but is also likely to detect additional rare OTUs. There is a positive overall correlation between cecal and fecal abundance as shown in Figure 4; this correlation is however negligible in the more dominant OTUs comprising over $10 \%$ of total sequences. This trend was found to be reproducible over the three trials that harbour very different microbial communities.

\section{Conclusions}

Based on the findings of the present study, we anticipate that with enough sequencing depth, fecal samples can be used to reflect the presence and absence of the vast majority of the members of the cecal community. However, the two communities are generally very distinct based on alpha and beta diversity. Positive correlation cannot be used to accurately predict OTU counts in the low abundance part of the microbiota due to it also carrying the highest number of unshared OTUs, nor in high abundance OTUs where correlation is minimal. However, regardless of community structure differences, i.e., species showing different abundance, dominance and equitability, both cecal and fecal microbiota analyses are likely to accurately report if a treatment or condition has induced changes in microbiota. This conclusion is based on the high number of shared species that represent $99.25 \%$ of all community members, which would be part of the community response to treatment. Although control/treatment differences could be detected using either cecal or fecal samples, conclusions drawn from the separate analyses are likely to differ. Hence choice of sampling site remains critical in experimental design as fecal microbiota do not provide a complete indication of cecal community structure.

\section{Methods}

\section{Chicken trials}

The bird trials were performed as previously described [18]. Briefly, male Cobb 500 broiler chickens were reared on feed comprised of wheat, soybean, barley, canola, peas, meat, tallow, limestone and vitamin mix with free access to food and water. Birds were euthanized by cervical dislocation on day 25 and samples collected for microbial analysis. Three independent replicate trials were performed. Samples were collected from an average 
of 70 birds from each trial. Cloacal (fecal) swabs were taken with sterile cotton swab inserted 10-12 mm into the cloacal opening and gently rotated to collect a sample of the fecal material. Cecal contents were collected by opening the birds immediately after euthanasia, cutting off one cecum, and manually squeezing content into a sterile tube. From one trial both ceca were harvested from 20 birds and content of each taken as separate samples. The swabs and cecal content were snap frozen on dry-ice and transported to the laboratory for processing.

\section{Ethics statement}

All animal work was conducted according to the national and international guidelines for animal welfare. The animal trials were approved and monitored by the Animal Ethics Committees of the University of Adelaide (Approval No. S-2010-080) and the Department of Primary Industries and Resources, South Australia (Approval No. 08/10).

\section{DNA preparation, sequencing, and data analysis}

DNA was prepared as described before, following the method detailed in $\mathrm{Yu}$ and Morrison [48]. The primers used to PCR amplify the V1-V3 region of the 16S rRNA gene were (forward primer [49], 5' AGAGTTTGATCCT GG 3'; reverse primer [50]), 5' TTACCGCGGCTGCT 3'). Both primers also included 454 sequencing regions and the series of forward primers included bar-code sequences to allow multiplexing of samples in a sequencing pool. Pyrosequencing was performed using a Roche/454 FLX Genome Sequencer according to the manufacturer's instructions. Sequences were analysed using PyroBayes [51], pintail chimera detection algorithm [52] and Qiime v1.6.0 [53]. Sequences were quality trimmed as described previously [48]. OTU picking was done at 3\% divergence level, de-noising error-correction, abundance and amplicon estimation using the USEARCH algorithm $[54,55]$. OTUs represented with less than 10 sequences and present in less than 5 samples were filtered out of the analysis. Samples represented by fewer than 1000 quality trimmed and filtered sequences were removed from the analysis and to maintain a paired data design the other sample of the rejected fecal/cecal sample pair was also removed, resulting in a total of 326 sequenced samples, from 163 birds, across three independent trials (52, 54 and 57 birds for trial 1 to trial 3 respectively), each bird represented with both fecal and cecal sample. Normalization of OTU tables was done by performing multiple rarefactions 100 times and averaging counts using a custom Perl script. OTUs were matched to their closest culturable isolate using EzTaxon [56]. R statistical software was used to inspect the correlation between cecal and fecal samples. The amplicon sequence data is available at MGRAST under accession number 4614960.3 (http://metagenomics.anl.gov/metagenomics.cgi? page=MetagenomeOver view\&metagenome $=4614960.3)$.

\section{Competing interests}

The authors declare that they have no competing interests.

\section{Authors' contributions}

MSG and RJH carried out the animal trials and DS, RJM, and HC performed the laboratory based work. DS analysed the data and drafted the manuscript. RJM supervised the project and contributed to the data interpretation. DS, MSG, RJH and RJM contributed to project conceptualization and revision of the manuscript. All authors read and approved the final manuscript.

\section{Acknowledgements}

This research was conducted within the Poultry CRC, established and supported under the Australian Government's Cooperative Research Centres Program. The data were analyzed using the Isaac Newton High Performance Computing (HPC) System at Central Queensland University; we wish to acknowledge continuous support in HPC computing provided by Jason Bell.

\section{Author details}

${ }^{1}$ Central Queensland University, School of Medical and Applied Sciences, Bruce Highway, Rockhampton, QLD 4702, Australia. ${ }^{2}$ Australian Animal Health Laboratory, CSIRO Animal, Food and Health Sciences, Geelong, VIC 3220, Australia. ${ }^{3}$ RMIT University, Poultry Cooperative Research Centre, University of New England Armidale, New South Wales 2315, Australia. ${ }^{4}$ South Australian Research and Development Institute, Pig and Poultry Production Institute, Roseworthy, South Australia 5371, Australia. ${ }^{5}$ The University of Adelaide, School of Animal and Veterinary Sciences Roseworthy, Roseworthy, South Australia 5371, Australia. ${ }^{6}$ University of South Australia, Research Office, Adelaide, South Australia 5001, Australia. ${ }^{7}$ RMIT University, Biotechnology and Ecological Biology, School of Applied Sciences, Bundoora, VIC 3083, Australia.

Received: 20 May 2014 Accepted: 16 February 2015

Published online: 27 February 2015

\section{References}

1. Clench MH, Mathias JR. The avian cecum: a review. Wilson Bull. 1995;107:93-121.

2. Mead GC. Microbes of the avian cecum: types present and substrates utilized. J Exp Zool Suppl. 1989;3:48-54.

3. Gasaway WC, White RG, Holleman DF. Digestion of dry matter and absorption of water in the intestine and cecum of rock ptarmigan. Condor. 1976;78:77-84.

4. Obst BS, Diamond JM. Interspecific variation in sugar and amino acid transport by the avian cecum. J Exp Zool Suppl. 1989;3:117-26.

5. Jozefiak D, Sip A, Rawski M, Rutkowski A, Kaczmarek S, Hojberg O, et al. Dietary divercin modifies gastrointestinal microbiota and improves growth performance in broiler chickens. Br Poult Sci. 2011;52:492-9.

6. Amerah AM, Peron A, Zaefarian F, Ravindran V. Influence of whole wheat inclusion and a blend of essential oils on the performance, nutrient utilisation, digestive tract development and ileal microbiota profile of broiler chickens. Br Poult Sci. 2011:52:124-32.

7. Siragusa GR, Haas GJ, Matthews PD, Smith RJ, Buhr RJ, Dale NM, et al. Antimicrobial activity of lupulone against Clostridium perfringens in the chicken intestinal tract jejunum and caecum. J Antimicrob Chemother. 2008;61:853-8.

8. Cressman MD, Yu Z, Nelson MC, Moeller SJ, Lilburn MS, Zerby HN. Interrelations between the microbiotas in the litter and in the intestines of commercial broiler chickens. Appl Environ Microbiol. 2010;76:6572-82.

9. Lin J, Hunkapiller AA, Layton AC, Chang YJ, Robbins KR. Response of intestinal microbiota to antibiotic growth promoters in chickens. Foodborne Pathog Dis. 2013;10:331-7.

10. Nakphaichit M, Thanomwongwattana S, Phraephaisarn C, Sakamoto N, Keawsompong S, Nakayama J, et al. The effect of including Lactobacillus reuteri KUB-AC5 during post-hatch feeding on the growth and ileum microbiota of broiler chickens. Poult Sci. 2011;90:2753-65.

11. Lee KW, Li G, Lillehoj HS, Lee SH, Jang SI, Babu US, et al. Bacillus subtilis-based direct-fed microbials augment macrophage function in broiler chickens. Res Vet Sci. 2011;91:e87-91.

12. Juricova H, Videnska P, Lukac M, Faldynova M, Babak V, Havlickova H, et al. Influence of Salmonella enterica serovar enteritidis infection on the development of the cecum microbiota in newly hatched chicks. Appl Environ Microbiol. 2013;79:745-7. 
13. Stanley D, Keyburn AL, Denman SE, Moore RJ. Changes in the caecal microflora of chickens following Clostridium perfringens challenge to induce necrotic enteritis. Vet Microbiol. 2012;159:155-62.

14. Lan PT, Sakamoto M, Benno Y. Effects of two probiotic Lactobacillus strains on jejunal and cecal microbiota of broiler chicken under acute heat stress condition as revealed by molecular analysis of $16 \mathrm{~S}$ rRNA genes. Microbiol Immunol. 2004;48:917-29.

15. Burkholder KM, Thompson KL, Einstein ME, Applegate TJ, Patterson JA. Influence of stressors on normal intestinal microbiota, intestinal morphology, and susceptibility to Salmonella enteritidis colonization in broilers. Poult Sci. 2008;87:1734-41.

16. Sergeant MJ, Constantinidou C, Cogan TA, Bedford MR, Penn CW, Pallen MJ. Extensive microbial and functional diversity within the chicken cecal microbiome. PLoS One. 2014;9:e91941.

17. Torok VA, Ophel-Keller K, Loo M, Hughes RJ. Application of methods for identifying broiler chicken gut bacterial species linked with increased energy metabolism. Appl Environ Microbiol. 2008;74:783-91.

18. Stanley D, Denman SE, Hughes RJ, Geier MS, Crowley TM, Chen H, et al. Intestinal microbiota associated with differential feed conversion efficiency in chickens. Appl Microbiol Biotechnol. 2012;96:1361-9.

19. Stanley D, Geier MS, Denman S, Haring VR, Crowley TM, Hughes RJ, et al. Identification of chicken intestinal microbiota correlated with the efficiency of energy extraction from feed. Vet Microbiol. 2013;164:85-92.

20. Sklan D, Shachaf B, Baron J, Hurwitz S. Retrograde movement of digesta in the duodenum of the chick: extent, frequency, and nutritional implications. J Nutr. 1978;108:1485-90.

21. Brummermann M, Braun EJ. Effect of salt and water balance on colonic motility of white leghorn roosters. Am J Physiol. 1995;268:R690-8.

22. Stanley D, Geier MS, Hughes RJ, Denman SE, Moore RJ. Highly variable microbiota development in the chicken gastrointestinal tract. PLoS One. 2013;8:e84290.

23. Pang W, Vogensen FK, Nielsen DS, Hansen AK. Faecal and caecal microbiota profiles of mice do not cluster in the same way. Lab Anim. 2012;46:231-6.

24. Sekelja M, Rud I, Knutsen SH, Denstadli V, Westereng B, Naes T, et al. Abrupt temporal fluctuations in the chicken fecal microbiota are explained by its gastrointestinal origin. Appl Environ Microbiol. 2012;78:2941-8.

25. Thomas VG. Nutritional, morphological, and behavioural considerations for rearing birds for release. J Orn. 1987;128:423-30.

26. Gong J, Forster RJ, Yu H, Chambers JR, Wheatcroft R, Sabour PM, et al. Molecular analysis of bacterial populations in the ileum of broiler chickens and comparison with bacteria in the cecum. FEMS Microbiol Ecol. 2002:41:171-9.

27. Zhu XY, Zhong T, Pandya Y, Joerger RD. $16 \mathrm{~S}$ rRNA-based analysis of microbiota from the cecum of broiler chickens. Appl Environ Microbiol. 2002;68:124-37.

28. Lu J, Idris U, Harmon B, Hofacre C, Maurer JJ, Lee MD. Diversity and succession of the intestinal bacterial community of the maturing broiler chicken. Appl Environ Microbiol. 2003;69:6816-24.

29. van der Wielen PW, Keuzenkamp DA, Lipman L, van Knapen F, Biesterveld S. Spatial and temporal variation of the intestinal bacterial community in commercially raised broiler chickens during growth. Microb Ecol. 2002:44:286-93.

30. Sokol H, Seksik P, Furet JP, Firmesse O, Nion-Larmurier I, Beaugerie L, et al. Low counts of Faecalibacterium prausnitzii in colitis microbiota. Inflamm Bowel Dis. 2009;15:1183-9.

31. Frank DN, St Amand AL, Feldman RA, Boedeker EC, Harpaz N, Pace NR. Molecular-phylogenetic characterization of microbial community imbalances in human inflammatory bowel diseases. Proc Natl Acad Sci U S A. 2007;104:13780-5.

32. Atarashi K, Tanoue T, Shima T, Imaoka A, Kuwahara T, Momose $Y$, et al. Induction of colonic regulatory $T$ cells by indigenous Clostridium species. Science. 2011;331:337-41.

33. Gong J, Si W, Forster RJ, Huang R, Yu H, Yin Y, et al. 16S rRNA gene-based analysis of mucosa-associated bacterial community and phylogeny in the chicken gastrointestinal tracts: from crops to ceca. FEMS Microbiol Ecol. 2007:59:147-57.

34. Giannenas I, Tontis D, Tsalie E, Chronis EF, Doukas D, Kyriazakis I. Influence of dietary mushroom Agaricus bisporus on intestinal morphology and microflora composition in broiler chickens. Res Vet Sci. 2010;89:78-84.

35. Salyers AA, Vercellotti JR, West SE, Wilkins TD. Fermentation of mucin and plant polysaccharides by strains of Bacteroides from the human colon. Appl Environ Microbiol. 1977;33:319-22.
36. Wrigley DM. Inhibition of Clostridium perfringens sporulation by Bacteroides fragilis and short-chain fatty acids. Anaerobe. 2004;10:295-300.

37. Round JL, Mazmanian SK. Inducible Foxp3+ regulatory T-cell development by a commensal bacterium of the intestinal microbiota. Proc Natl Acad Sci U S A. 2010;107:12204-9.

38. Louis P, Flint HJ. Diversity, metabolism and microbial ecology of butyrate-producing bacteria from the human large intestine. FEMS Microbiol Lett. 2009;294:1-8.

39. Morrison M, Miron J. Adhesion to cellulose by Ruminococcus albus: a combination of cellulosomes and Pil-proteins? FEMS Microbiol Lett. 2000;185:109-15.

40. Kaakoush NO, Sodhi N, Chenu JW, Cox JM, Riordan SM, Mitchell HM. The interplay between Campylobacter and Helicobacter species and other gastrointestinal microbiota of commercial broiler chickens. Gut Pathog 2014;6:1841.

41. Thompson CL, Mikaelyan A, Brune A. Immune-modulating gut symbionts are not "Candidatus Arthromitus". Mucosal Immunol. 2013;6:200-1.

42. Gaboriau-Routhiau V, Rakotobe S, Lecuyer E, Mulder I, Lan A, Bridonneau C, et al. The key role of segmented filamentous bacteria in the coordinated maturation of gut helper T cell responses. Immunity. 2009;31:677-89.

43. Ivanov II, Atarashi K, Manel N, Brodie EL, Shima T, Karaoz U, et al. Induction of intestinal Th17 cells by segmented filamentous bacteria. Cell. 2009;139:485-98.

44. Umesaki Y, Okada Y, Matsumoto S, Imaoka A, Setoyama H. Segmented filamentous bacteria are indigenous intestinal bacteria that activate intraepithelial lymphocytes and induce MHC class II molecules and fucosyl asialo GM1 glycolipids on the small intestinal epithelial cells in the ex-germ-free mouse. Microbiol Immunol. 1995;39:555-62.

45. Talham GL, Jiang HQ, Bos NA, Cebra JJ. Segmented filamentous bacteria are potent stimuli of a physiologically normal state of the murine gut mucosal immune system. Infect Immun. 1999;67:1992-2000.

46. Thompson CL, Vier R, Mikaelyan A, Wienemann T, Brune A. 'Candidatus Arthromitus' revised: segmented filamentous bacteria in arthropod guts are members of Lachnospiraceae. Environ Microbiol. 2012;14:1454-65.

47. Ivanov II, Littman DR. Segmented filamentous bacteria take the stage. Mucosal Immunol. 2010;3:209-12.

48. Yu Z, Morrison M. Improved extraction of PCR-quality community DNA from digesta and fecal samples. Biotechniques. 2004;36:808-12.

49. Lane D J. 16S/23S rRNA sequencing. In: Stackebrandt E, Goodfellow M, editors. Nucleic acid techniques in bacterial systematics. Chichester, United Kingdom: John Wiley and Sons; 1991. pp. 115-175.

50. Felske A, Rheims H, Wolterink A, Stackebrandt E, Akkermans AD. Ribosome analysis reveals prominent activity of an uncultured member of the class Actinobacteria in grassland soils. Microbiology. 1997;143:2983-9.

51. Quinlan AR, Stewart DA, Stromberg MP, Marth GT. Pyrobayes: an improved base caller for SNP discovery in pyrosequences. Nat Methods. 2008;5:179-81.

52. Ashelford KE, Chuzhanova NA, Fry JC, Jones AJ, Weightman AJ. At least 1 in $2016 \mathrm{~S}$ rRNA sequence records currently held in public repositories is estimated to contain substantial anomalies. Appl Environ Microbiol. 2005;71:7724-36

53. Caporaso JG, Kuczynski J, Stombaugh J, Bittinger K, Bushman FD, Costello EK, et al. QIIME allows analysis of high-throughput community sequencing data. Nat Methods. 2010;7:335-6.

54. Edgar RC. Search and clustering orders of magnitude faster than BLAST. Bioinformatics. 2010;26:2460-1.

55. Edgar RC, Haas BJ, Clemente JC, Quince C, Knight R. UCHIME improves sensitivity and speed of chimera detection. Bioinformatics. 2011;27:2194-200.

56. Chun J, Lee JH, Jung Y, Kim M, Kim S, Kim BK, et al. EzTaxon: a web-based tool for the identification of prokaryotes based on 16S ribosomal RNA gene sequences. Int J Syst Evol Microbiol. 2007;57:2259-61. 